Sección monográfica / Monographic section

\title{
Los últimos y las últimas de la cadena. Calidad y trabajo en el sector citrícola valenciano
}

\section{The last in the chain. Quality and work in the Valencian citrus sector}

\author{
Francisco Torres Pérez* \\ Universidad de Valencia, España. \\ francisco.torres@uv.es
}

\section{Yaiza Pérez Alonso (iD}

Universidad de Valencia, España.

yaiza.perez@uv.es

Recibido / Received: 14/04/2020

Aceptado / Accepted: 28/09/2020

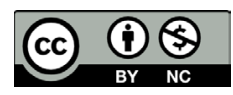

\section{RESUMEN}

Los trabajadores y trabajadoras agrícolas ocupan la última posición en las cadenas globales agroalimentarias. Éstas, hegemonizadas por las grandes distribuidoras, trasladan de arriba a abajo una creciente presión competitiva en la que la calidad es un elemento central. Este artículo aborda las consecuencias que los requerimientos de calidad tienen sobre la mano de obra en La Ribera del Xúquer (Valencia), un territorio citrícola de exportación. Nuestros resultados indican que los estándares de calidad no suponen una mayor calidad de trabajo en el campo, aumentan las diferencias entre trabajadores y refuerzan la segmentación étnica. En los almacenes, tradicionalmente feminizados, los requerimientos de calidad si bien suponen una mayor polivalencia y control del espacio, comportamiento y cuerpo de la trabajadora, han contribuido a un cumplimiento más estricto del convenio colectivo.

Palabras clave: cadenas agroalimentarias; calidad; trabajo; sector cítrico.

\begin{abstract}
Agricultural workers occupy the last position in the global agri-food chains. These, hegemonized by the large distributors, transfer from top to bottom an increasing competitive pressure in which quality is a central element. This paper tackles the consequences of quality norms on the workforce in La Ribera del Xúquer (Valencia), a citrus exporting territory. Our results show that quality standards do not imply a higher quality of work in the field, increase differences between workers and reinforce ethnic segmentation. In the warehouses, traditionally feminized, the quality requirements, although they imply greater versatility and control of the worker's space, behaviour and body, have contributed to stricter compliance with the collective agreement.
\end{abstract}

Keywords: agri-food chains; quality labour; citrus sector.

\footnotetext{
*Autor para correspondencia/ Corresponding author: Francisco Torres, francisco.torres@uv.es 


\section{INTRODUCCIÓN}

Desde finales del siglo XX, la calidad constituye un elemento central en la configuración, estratificación y funcionamiento de las cadenas globales agroalimentarias. Así la calidad se ha convertido en un modelo de gobernanza empresarial (Ponte, Gibbon y Vestergaard, 2011), gestionado por una compleja estandarización (Gibbon, Bair y Ponte, 2008), pública y sobre todo privada, con el objetivo de coordinar las actividades de los diferentes actores de la cadena, hacerlo just-in-time y de acuerdo con los cambiantes gustos del mercado global. Hoy, la calidad es un aspecto central de la posición que se ocupa en el mercado global y afecta a la producción, manipulación, comercialización y distribución. Las cadenas globales agroalimentarias, hegemonizadas por las grandes exportadoras y distribuidoras, trasladan de arriba a abajo en particular sobre los actores que ocupan la última posición en la cadena, productores locales y trabajadores y trabajadoras, una creciente presión competitiva en la que la calidad es un requerimiento central.

En este artículo, nos focalizamos en los impactos de la calidad sobre las condiciones de trabajo en el sector de los cítricos en La Ribera del Xúquer, una comarca natural e histórica, en la provincia de Valencia, dedicada desde mediados del siglo XIX, al cultivo intensivo de cítricos orientado a la exportación. Dada la crisis de la citricultura en las dos últimas décadas, provocada por la bajada sistemática de precios en origen, se ha dado una progresiva sustitución de cítricos por caqui con mayor rentabilidad media, aunque con fuerte descenso en los últimos años. Este cultivo tiene la misma organización de trabajo que los cítricos.

Este artículo aborda las implicaciones de los estándares y normas de calidad en los procesos de trabajo, tanto en el campo como en los almacenes de procesado, en estas comarcas valencianas plenamente insertas en las cadenas agroalimentarias globales. Nuestro objetivo es triple. En primer lugar, analizar si los sistemas de gestión de calidad han contribuido o no a mejorar la calidad del trabajo. En segundo lugar, dado que el recurso a la mano de obra inmigrante es estructural, estudiar si tiende a reforzar o no la segmentación étnica del mercado de trabajo. En tercer lugar, captar sus efectos desde el punto de vista del sistema sexo-género en este mercado de trabajo. Nuestro análisis se basa en los resultados obtenidos en el marco del Proyecto CSO2017-85507-P. "Gobernanza de la calidad en las cadenas globales agroalimentarias. Un análisis comparado de los territorios agro-exportadores en España". Se ha realizado trabajo de campo entre octubre de 2018 y febrero de 2020 , con observación, entrevistas informales y entrevistas individuales y grupales transcritas a 29 informantes clave (trabajadores y trabajadoras, jefes de cuadrilla y encargadas de almacén, técnicos de calidad, sindicalistas e inspectores de trabajo)'. Igualmente, se ha trabajado con el Censo Agrario 2009, el último disponible, y los datos de LABORA Servei Valencià d'Ocupació i Formació.

\section{CADENAS AGROALIMENTARIAS GLOBALES, CALIDAD, TERRITORIOS Y RELACIONES DE PRODUCCIÓN}

En las últimas décadas, las cadenas agroalimentarias globales presentan una serie de tendencias comunes. Aumentan su extensión y penetración en los territorios, así como la posición de dominio de las grandes empresas exportadoras y distribuidoras. Desde el punto de vista de las cadenas globales de mercancías, las cadenas agroalimentarias serían

\footnotetext{
1 De estos informantes, 17 son hombres y 12 mujeres, 17 españoles y 12 inmigrantes (de origen rumano, búlgaro, polaco, marroquî y ecuatoriano). Las entrevistas se realizaron en castellano y valenciano y se presentan como EIno, las entrevistas individuales, y EGno, las grupales.
} 
el paradigma de cadena dirigida y controlada por el comprador, aunque este control no es total dado que intervienen una diversidad de actores: empresas, intermediarios, sindicatos, gestores públicos... (Gereffi, Humphrey y Sturgeon, 2005). Coordinar la acción de esta pluralidad de actores, en diferentes escalas territoriales y respondiendo a los variables requerimientos del mercado global, constituye una tarea muy compleja y central. En las últimas décadas, la calidad se ha convertido en un modelo de gobernanza empresarial (Carton y Lara, 2010; Ponte et al., 2011); estas normas de calidad se establecen por medio de la estandarización (Gibbon et al., 2008).

Estas tendencias globales se concretan de forma relativamente diferenciada según los contextos estatales y locales, como ocurre en otros ámbitos de la globalización (Robertson, 1995; Barañano, 2005). Por un lado, las cadenas globales se insertan en territorios con una estructura social, política e institucional, que constituye el marco de esa inserción al mismo tiempo que, con su presencia, lo modifica. Aquí destacamos la relevancia analítica de las relaciones de producción, las formas concretas de competencia capital-trabajo, y de las relaciones de reproducción social, para captar "el modo en que se reproducen las posiciones vulnerables" de la mano de obra (Pedreño, 2014, p. 23). Por otro lado, los productores locales, las empresas, los sindicatos y la propia Administración intervienen también, con posiciones desiguales de poder, en la definición concreta de los lazos que vinculan estos territorios a las cadenas globales, regidas por estándares de calidad.

Si la calidad es una construcción social, los estándares de calidad remiten a un proceso cambiante y complejo en el que participan diferentes actores, con distintas posiciones y poder. Aunque la introducción de estos estándares en el sector agroalimentario es relativamente reciente (Castro, Moraes y Cutillas, 2017), ya tiene un considerable desarrollo. En la actualidad, una empresa exportadora debe cumplir con una normativa estatal de creciente relevancia ${ }^{2}$. Además, debe cumplir con las regulaciones supranacionales de la Unión Europea y de los Tratados de Libre Comercio firmados. Por último, pero decisivo, debe ajustarse a los protocolos de calidad exigidos por las grandes distribuidoras, que constituyen la llave de acceso al mercado global (Moraes y Cutillas, 2014). Entre los estándares más relevantes en la horticultura, presentes en nuestra zona de estudio, podemos señalar GlobalGAP, el más generalizado, BCR (British Retail Consortium), para cadenas británicas, IFS (Food International Featured Standard), para cadenas alemanas y francesas, ISO (International Organization for Standardization), entre otros.

Algunos actores de esta red regulatoria global no son nuevos, como la administración en Europa Occidental y Estados Unidos. Otros actores, privados, surgen en los años 90 del siglo pasado con la globalización neoliberal. En efecto, los certificados de calidad remiten a un complejo entramado de propietarios de los estándares, entidades de certificación, consultoras, etc., con una gran presencia de las empresas transnacionales dándose una situación que Baylos (2009) califica de "autorreferencialidad", los actores con mayores intereses controlan el conjunto de normas, y Pries (2008) de merma del poder regulatorio del Estado.

El contenido de estas normas y protocolos, denominadas Buenas Prácticas Agrícolas, es variado, pero pueden ordenarse en cuatro ámbitos: trazabilidad y registro del proceso; insumos fitosanitarios, uso del agua; almacenamiento y manipulación; y aspectos relacionados con la salud y protección de los trabajadores (Moraes y Cutillas, 2014). Este último aspecto, referente a las condiciones de trabajo, aunque no está presente en todos los protocolos sí ha aumentado su presencia. En América Latina, con normas derivadas de la Decent Work Agenda de la OIT (Carton y Lara, 2010; Selwyn, 2013); en el caso europeo, el módulo GRASP de GlobalCAP (Global Risk Assessment on Social Practices) es el más presente.

2 En esa línea, puede destacarse la Agencia Española de Consumo, Seguridad Alimentaria y Nutrición y la Ley 28/2015, de 30 de julio, para la defensa de la calidad alimentaria. 
Sobre la creciente relevancia de la calidad a nivel productivo, económico y comercial, contamos con una amplia literatura. Sin embargo, esta es más escasa respecto a sus impactos en las condiciones de trabajo (Selwyn, 2013; Castro et al., 2017). Diversos estudios en México, Argentina y Brasil destacan como los estándares de calidad no han mejorado las condiciones de trabajo y vida, incluso las han precarizado (Carton y Lara, 2010; Quaranta, 2009; Bonanno y Cavalcanti, 2012). Un balance similar, con las lógicas diferencias, ofrecen Castro et al. (2017) para la uva de mesa en Murcia. En otros estudios se señalan situaciones más positivas para los trabajadores. En Argentina y Uruguay, estas mejoras se derivan, no tanto de los estándares de calidad de las cadenas globales sino de la legislación laboral, la fiscalización administrativa y la presencia sindical (Quaranta, 2009; Moraes, 2011). Para los casos de Ecuador-Chiquita y Sao Francisco, en el Nordeste de Brasil, Striffler (2008) y Selwyn (2013) destacan el factor de la presencia sindical. En términos de Selwyn (2013), la capacidad de los trabajadores "para transformar su poder estructural en poder asociativo para extraer concesiones al capital" (p. 77).

\section{LA RIBERA DEL XÚQUER COMO ÁREA CITRÍCOLA VALENCIANA}

La Ribera del Xúquer es una comarca natural e histórica, articulada por el río Xúquer, y que agrupa las comarcas administrativas de la Ribera Alta y la Ribera Baixa, en la provincia de Valencia. Esta comarca de rica agricultura se dedica desde mediados del siglo XIX, al cultivo intensivo de cítricos para exportación. Con los lógicos altibajos en más de un siglo, los buenos precios y la ausencia de competidores en el mercado europeo proporcionaron importantes beneficios, permitió la acumulación de capital para la industrialización y modernización, así como una renta modesta pero relevante a sectores de agricultores (Bono, 2010).

Figura 1. Distribución porcentual de las explotaciones agrarias por tamaño según la titularidad de la explotación. La Ribera del Xúquer.

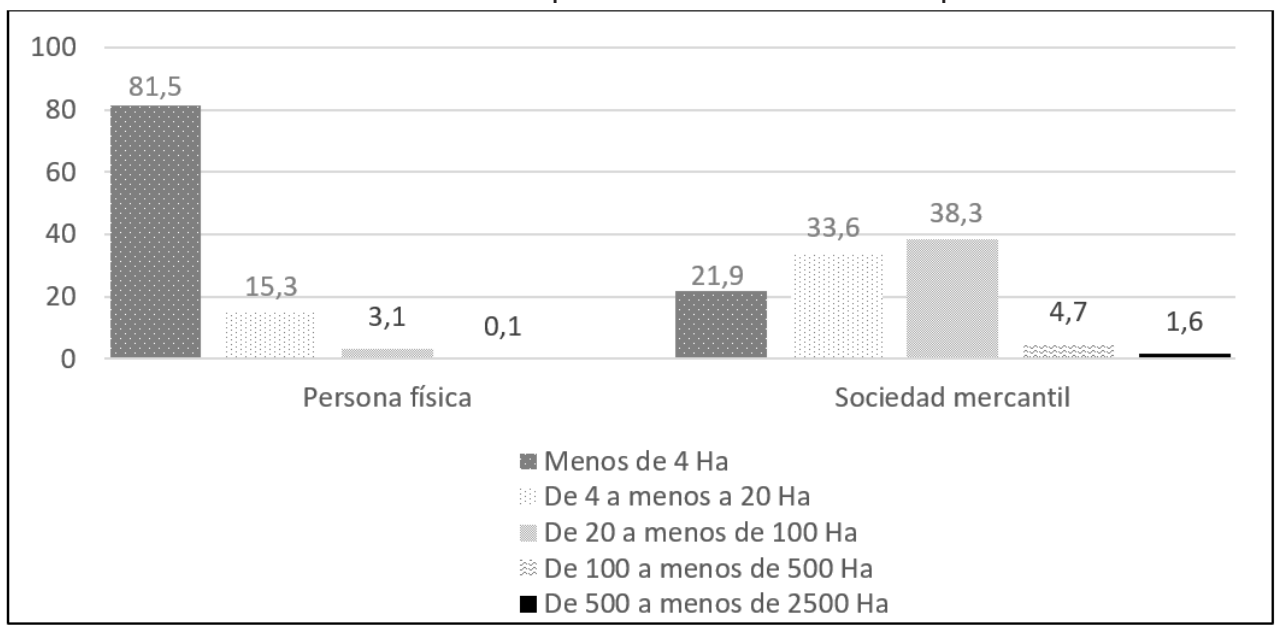

Fuente: elaboración propia a partir de Censo Agrario (2009).

Desde los años setenta, la Ribera del Xúquer tiene una estructura económica diversificada, con un mayoritario sector servicios y una relevante industria, aunque la 
citricultura conserva su importancia económica y social. A pesar de los grandes cambios económicos y sociales, se mantienen los rasgos que definen la zona si bien transformados. Como en el pasado, se trata de una estructura de pequeños propietarios, el $81,5 \%$ de los cuales tiene una explotación menor de $4 \mathrm{Ha}$. (figura 1). A diferencia del pasado, una gran parte son agricultores a tiempo parcial. Asimismo, se mantiene la relevancia de las cooperativas que, si bien comercializan sobre un tercio de la producción, han visto su posición debilitada ante la creciente hegemonía de las grandes cadenas. En el pasado, el comercio exterior estaba gestionado por empresas de capital valenciano (Gallego y Lamanthe, 2011). Desde los años noventa, se da una progresiva inserción de las grandes empresas y plataformas agroalimentarias en La Ribera, reforzando la integración vertical de la cadena citrícola global y la creciente estandarización.

Desde primeros del siglo XXI asistimos a la "crisis" de la citricultura valenciana, con la caída de precios en origen como consecuencia, entre otros, de dos factores. Por un lado, el incremento de la oferta de países terceros, particularmente de Marruecos y Sudáfrica. Por otro lado, la situación de oligopolio de un reducido número de grandes cadenas que controlan el mercado global (Gallego y Lamanthe, 2011; Caballero et al., 2012; Garrapa, 2017). Como alternativa, se ha dado una progresiva sustitución de cítricos por caqui dado su mayor rentabilidad promedio, aunque si bien en los últimos años ésta ha descendido. Este proceso ha sido muy relevante en La Ribera, con la creación en 1998 de la Denominación de Origen "Kaki Ribera del Xúquer", que en 2016 concentraba el 63\% del cultivo nacional (Vendrell, 2017).

\section{El mercado de trabajo en La Ribera}

Como sucede en otras zonas agrícolas, en La Ribera encontramos un mercado de trabajo etno-fragmentado. En términos de Piore (1983) tenemos dos situaciones tipo. En el mercado primario con contratos fijos, sueldos medios y buenas condiciones laborales, encontramos directivos, técnicos de calidad y personal administrativo cualificado, hombres y mujeres españoles. En el mercado secundario, el más numeroso en efectivos, los trabajadores y trabajadoras, fijos discontinuos, temporales y/o trabajadores de ETT, con escasa paga y trabajos penosos, que realizan las tareas en el campo, hombres inmigrantes en su mayoría, o en almacén, mujeres españolas. En este artículo nos centramos en este mercado secundario de trabajo.

En La Ribera, tanto en cítricos, caqui, como en otros cultivos (fruta de hueso), se mantiene el sistema tradicional de venta y recolección, aunque modificado. El agricultor "vende en el árbol" la cosecha por kilos al corredor o comercial que asume la responsabilidad de la recolección para lo que cuenta con cuadrillas de recolectores dirigidos por jefes de cuadrilla. En el pasado, el corredor estaba contratado por una cooperativa o empresa, así como, desde los años ochenta, los miembros de las cuadrillas. Los jornaleros eran vecinos del mismo municipio o colindante, hombres en su inmensa mayoría, muchos de ellos pequeños propietarios que necesitaban esos ingresos para la supervivencia familiar. El sistema funcionaba con un reparto de roles estrictamente diferenciado. Al mismo tiempo, su incrustación en el marco social de los pueblos y en las relaciones de parentesco, amistad o vecindad, entre corredors, jefes de cuadrillas y jornaleros, minimizaba las tensiones, tanto las demandas de los jornaleros como los abusos respecto a la norma establecida (Cucó, 1982, p. 272 y ss.).

Desde mediados de los años 90 , el sistema se enfrentaba a un doble reto. Por un lado, el abandono de los trabajadores españoles y la falta de mano de obra. Por otro lado, la creciente integración de La Ribera en la cadena agroalimentaria global y la presión competitiva para reducir costes. Como en otras zonas agroexportadoras españolas, 
la solución fue el recurso estructural de los trabajadores inmigrantes y el creciente protagonismo de las ETT.

Todo ello ha tenido consecuencias. Si bien las cooperativas mantienen la contratación de las cuadrillas, entre las empresas solo lo hacen una minoría de medias y grandes empresas, dedicadas a calidad Premium. La tendencia creciente es a la externalización a una ETT, con una clara degradación de las condiciones laborales, como luego veremos. Por otro lado, esta diversidad de actores (corredor, jefes de cuadrilla, trabajadores) ya no comparten, como en el pasado, una trama de relaciones sociales que establecía ciertos controles frente a los abusos. Estos controles desaparecieron con la trama social que los sustentaba. Hoy el marco social es otro, globalizado, individualista y neoliberal.

El paso previo a la comercialización de la naranja recolectada es su selección, preparación y empaquetado, en los almacenes. En el pasado y en la actualidad son las mujeres de estos pueblos, hijas y/o esposas de pequeños propietarios y trabajadores, quienes trabajan en los almacenes de forma estacional. Actualmente, la gran mayoría de las mujeres que trabajan en los almacenes son españolas, con una minoría de origen inmigrante.

\section{RECOLECCIÓN, CALIDAD Y CONSECUENCIAS PARA LOS TRABAJADORES Y TRA- BAJADORAS}

Con la introducción de nuevas variedades tempranas y tardías, la temporada de recolección se ha ampliado a casi 8 meses al año, con mayor intensidad en los meses de invierno. La recolección de la naranja o del caqui la realizan, en su gran mayoría, trabajadores inmigrantes entre los que destacan europeos del este, búlgaros y rumanos, latinos de distintas nacionalidades, marroquíes y pakistaníes. La crisis de 2008 cortó la fuga constante de trabajadores españoles; en la actualidad, se mantiene una minoría de recolectores españoles.

En la recolección participan hombres y, de forma minoritaria pero relevante, mujeres inmigrantes. Se tratan de mujeres europeas del Este y, en mucha menor medida, alguna marroquí. En muchos casos la mujer tiene una relación de parentesco y/o amistad con algún hombre de la cuadrilla.

Como antaño, la recolección se organiza por cuadrillas de trabajadores bajo la dirección del jefe de cuadrilla. Nuestros entrevistados y entrevistadas tuvieron su primer contacto con su cuadrilla actual por un "conocido", por haber coincidido en el pasado con alguno de sus miembros, "por Internet, por mil anuncios" o por haber recurrido a la misma ETT. En la actualidad, las ETT son la puerta de entrada al campo; más tarde, con mayores relaciones y experiencia, se puede ser contratado por una empresa o cooperativa.

“I1. Eh, el sistema para buscar trabajo, hoy en día, si no sabes un conocido, no entrarás

13. Todo enchufe

11. Solamente si tienes suerte, que sería un porcentaje muy bajo, el 1 o el $2 \%$

15. Es que ahora las empresas mismo te envían, tú dices ¡mira vas a dejar el currículo! Dicen, "no, mira, entra esta ETT, y ves allá a dejarlo ... Ellos mismos te envían porque ellos recogen gente de ahí" (recolectores búlgaros. EG04)

En La Ribera las cuadrillas más habituales suelen ser del mismo origen. Los españoles se agrupan en cuadrillas de españoles, que pueden incluir a algún inmigrante. Entre las 
cuadrillas de inmigrantes son muy frecuentes las del mismo origen: búlgaro, rumano, marroquí u otro; también son habituales cuadrillas multiculturales formadas por trabajadores de distintos orígenes.

El reparto de tareas de una cuadrilla es sencillo; todos sus componentes recolectan la naranja o el caqui de acuerdo con las indicaciones, depositan sus cajones en un sitio y cargan el camión. En muchas cuadrillas esta última tarea es rotatoria; en otras, nos dicen, lo hacen los jóvenes y, se dan casos de discriminación, como adjudicar sistemáticamente la carga de lo recogido a los miembros subsaharianos de la cuadrilla. En el caso de las cuadrillas mixtas, la o las mujeres cortan y recolectan como uno más, pero suele ser el hombre con el que tiene relación, novio, primo, amigo, quién saca su cajón fuera del campo y realiza su turno de carga.

Como otras figuras de intermediarios laborales, el jefe de cuadrilla recluta a sus miembros, dirige y controla su trabajo, registra lo recolectado, horas, etc., y es el responsable de la recolección ante el corredor o, en menor medida, ante la cooperativa o empresa directamente. En ocasiones, los jefes de cuadrilla hacen también de transportistas.

En la actualidad, el papel del jefe de cuadrilla está determinado por la reestructuración del sector, con la introducción de las grandes cadenas y los cambios en la trama social de la zona, los requerimientos de calidad y la creciente relevancia de las ETT (con diferencias entre jefes de cuadrilla de empresas y cooperativas y de ETT, que luego se abordan). Referente a calidad, el jefe de cuadrilla debe velar porque se cumplan los requerimientos en cuanto al calibre, tamaño, madurez y apariencia u otros, que le transmite el corredor y/o el técnico de calidad de la empresa o cooperativa. Según un técnico de calidad entrevistado, en un "buen funcionamiento de la cadena de calidad" (EI05), el jefe de cuadrilla debe transmitir los aspectos relevantes del fruto y/o de las parcelas que está recolectando, va "categorizando" el producto que se está recolectando y "validándolo" para que en el almacén se prepare un tratamiento u otro.

“Pero para mí el control (de calidad) empieza ya con el cabo de cuadrilla validando si la cosecha está agravada por alguna enfermedad o tiene muchos problemas de destrío, o cualquier cosa, toda esa categorización en campo llega a la entrada (del almacén) ... esa categorización se tiene que respetar, tanto a la hora de manipular, como logísticamente. Con el caqui la categorización en campo aún es mayor ¿Por qué? Recolectamos caqui desde principios de octubre hasta final de diciembre, y bueno, debido al gran volumen, pues es importante tener las categorías... estamos hablando, por ejemplo, parcelas de caqui que tienen salinidad, ... una maduración prematura y calibres más ajustados, entonces, la vida comercial del fruto es mucho menor que un caqui sin exceso de sales... Otra categoría, por ejemplo, tenemos una plaga muy importante en el caqui que es la mosca blanca que genera negrilla, eso también lo categorizaríamos porque habría que volcarlo al final del día y habría que aumentar la potencia y parámetros de lavado" (técnico calidad cooperativa El05).

En la actualidad, hay jefes de cuadrilla españoles y extranjeros de diversos orígenes. Se tratan de hombres, en su inmensa mayoría, aunque también hay algunas mujeres inmigrantes, europeas del Este, que trabajan como jefa de cuadrilla (de hombres). En el pasado, el jefe de cuadrilla era un trabajador con experiencia, que "sabe escribir... que sea responsable" (Cucó, 1982, p. 290). En la actualidad, entre los jefes de cuadrilla encontramos una amplia diversidad que, básicamente, se corresponde con las diferencias entre las cuadrillas de cooperativas, de empresas y de ETT. Entre las dos primeras categorías se llega a ser jefe de cuadrilla por trayectoria laboral, saber obrero acumulado y confianza del corredor. 
"Ser capataz es... que te respeten y hacerte tú respetar, ¿sabes? Si uno no está conforme, decirle: "Bueno, hasta aquí has llegado" Si la cosa va bien, va bien... pero hay gente... españoles... Hablo de españoles porque es lo que yo visto... sin responsabilidad... (Eso es) hacer la faena como te la mandan, porque yo he estado hasta escogiéndoles la naranja buena y mala" [jefe de cuadrilla, español, jubilado. El01]

Entre los jefes de cuadrilla de las ETT, aunque también hay una amplia diversidad, se dan casos de trabajadores y trabajadoras contactados por una ETT, para que monten una cuadrilla, en función de sus necesidades. En diversas ocasiones, la oferta implica también que su sueldo salga del de sus trabajadores (por el dinero del transporte o un tanto que se queda por cajón).

"Me dijeron: ¿Por qué tú no lo haces esto? Tú sabes muy bien el español, sabes moverte, podías ir al almacén y me dijeron a qué almacén tengo que ir, uno de Xeraco, ... busca una cuadrilla de diez personas, nosotros te vamos a decir dónde tienes la faena, van con sus coches [...] La gasolina no se paga a ellos... [...] por ejemplo, por cajón ... lo pone a 10 euros. Este dice: "10 euros, yo lo doy a 8" [...] Ya se queda dos... ya tienes dinero... tiene que ir todo en negro [...] me lo dijeron. "Tú no vas a hacer nada, tú entras al campo y ves a los que trabajan. Tú vas a estar, por ejemplo, con una báscula y mirar"

No te gustó ese rollo

Pues no. No, yo no soy capaz, ¿sabes? No, no lo soy. Podía aprovecharme, podría aprovecharme porque muchos no pueden traducir a algunas (trabajadoras). Yo no cobro dinero por eso" (trabajadora polaca de almacén, anteriormente en campo. El17)

En la recolección de naranja se trabaja a destajo, cobrándose por kilos recolectados, o a jornal, abonándose las horas trabajadas. El convenio de cítricos permite ambas modalidades. En la actualidad, la inmensa mayoría trabaja a destajo. Históricamente, los trabajadores de la zona han preferido el destajo, ya que comportaba un mayor sueldo; en las últimas dos décadas, las empresas y las ETT han generalizado el sistema. Con la bajada del precio del kilo de naranja o del caqui en origen y la reducción de la cláusula de garantía del convenio ${ }^{3}$, la modalidad de destajo tiende a bajar los costos laborales. Entre los trabajadores hay opiniones contrapuestas, quién prefiere el destajo porque considera que le beneficia (el rendimiento en destajo también depende del tipo de campo, de los árboles, etc.). Otros trabajadores preferirían ir a jornal y decimos preferirían porque no se les ofrece la opción.

En la recolección, se han generalizado los requisitos respecto a calibre y aspecto, a pesar de que se recolecta a destajo en la gran mayoría de casos. No se paga más pero se exige una selección del fruto. El ritmo que implica el destajo, para obtener el máximo de kilos, cuadra mal con la selección exigida. Además, como medida disciplinaria, se puede descontar y muchas veces se hace los kilos de frutos recolectados que no tienen "valor comercial" (no se ajusta a lo demandado).

Si bien esta es la situación más general, tenemos una heterogeneidad que correlaciona con los niveles de calidad y el tipo de trabajadores. En una minoría de empresas, medias y grandes, que trabajan calidades Premium con campos propios o de agricultores tutelados, la primera pasada de la recolección de naranja la realizan a jornal trabajadores fijos discontinuos, con requerimientos muy estrictos; ya la segunda pasada y posterior, si la hubiere, se hace a destajo. En el caso de las cooperativas es habitual que se combine jornal y destajo, según los pedidos, y la mayoría de trabajadores son, también, fijos discontinuos. Sin embargo, en la gran mayoría de empresas y en todas las ETT la recolección se hace a

3 En el anterior convenio de cítricos se establecía que en la modalidad de destajo se cobraría como mínimo el equivalente a las horas trabajadas. En el actual, se establece un salario "correspondiente a lo realmente recolectado con un mínimo de 9 euros por hora trabajada" (art.9), cuando el mismo convenio establece un salario hora de 10,44 euros en la actual campaña. Convenio colectivo de recolección de cítricos. Comunidad Valenciana. 2018-2022. DOGV 8578/26.06.2019. 
destajo a cargo de fijos discontinuos, temporales y, sobre todo, trabajadores de ETT. A su vez, como veremos posteriormente, esta tipología también correlaciona con el grado de irregularidades laborales.

“Normalmente trabajáis a destajo o a horas, o por horas?

13. A destajo

X. Ahora no trabaja... ni el 5\% de toda la gente a horas (jornal)

11. En M. N. y F. (empresas), trabajan a horas

X. En M. N. trabajan a horas los fijos discontinuos

11. Vale

15. Y las primeras semanas

X. En lo del resto de las ETT que van para M. N., como vosotros... a destajo

15. Yo prefiero trabajar a destajo

11. Yo también

15. Porque saco más

X. ¡A ver, a ver, el destajo! Mira, eh... todos los campos no son iguales, no tienen la misma producción, el camión está a medio kilómetro" (recolectores rumano, marroquí y español. EG03)

Los sueldos varían de acuerdo con la tipología de empresas y trabajadores antes esbozada, con una alta variabilidad. En el caso de las empresas que "trabajan calidad y cumplidoras" (sindicalista. El08) y las cooperativas, los trabajadores fijos discontinuos pueden obtener un sueldo mensual entre 1000 euros, trabajando de lunes a viernes, y 1400 euros netos, trabajando sábados. Entre estos fijos discontinuos la mayoría son trabajadores españoles. Las mismas jornadas de trabajo reportan entre 650-700 y 800 euros netos, de media, para los trabajadores de ETT, en su inmensa mayoría inmigrantes.

Una nota particular merece el sueldo del jefe de cuadrilla. Una parte de las empresas y las cooperativas aplican el convenio, que prevé en la modalidad a jornal un suplemento de un euro más por hora. Sin embargo, la inmensa mayoría de las cuadrillas trabajan a destajo. En este caso, se cobra sobre los kilos recolectados. Tradicionalmente, el jefe de cuadrilla participaba como uno más en el reparto de lo recolectado y el corredor, la empresa o cooperativa, le pagaban un plus. Este sistema se mantiene para una parte de los jefes de cuadrilla. Sin embargo, con la entrada de las ETT en la zona muchas de éstas dejaron de pagar el plus al jefe de cuadrilla, ejemplo que fue imitado por no pocas empresas. Dicho de otra forma, en el estrato más precarizado del trabajo, si el jefe de cuadrilla quiere obtener un plus respecto a sus trabajadores debe recurrir a vías irregulares, como cobrar por el transporte, o quedarse con un tanto de cajones recogidos, además de su parte correspondiente de lo recolectado.

Respecto a irregularidades, una de las comunes en 2012-2014 era trabajar sin contrato ni alta de Seguridad Social (Garrapa, 2017). No es esa la situación en la actualidad, dadas las campañas de inspección de trabajo, particularmente intensa en 2015 y 2016, y como refleja la figura 2, la inmensa mayoría trabaja con contrato. Las irregularidades más frecuentes son el cobro efectivo de un salario inferior al que establece el convenio y el fraude en los días de trabajo declarados, inferiores a los reales, y en las cotizaciones derivadas. La dinámica es la siguiente: se recolecta a destajo y, dado el bajo precio del cajón recolectado, el sueldo resultante es inferior al establecido en convenio. Esta situación se enmascara 
mediante nóminas por la misma cantidad, pero declarando menos días trabajados para que el sueldo se ajuste a lo mínimo establecido. Estas nóminas, formalmente correctas, son las que se presentan ante la administración, lo que dificulta enormemente la acción de inspección de trabajo.

Figura 2. Evolución del número de contratos en el sector de la agricultura. La Ribera del Xúquer. 2009-2019.

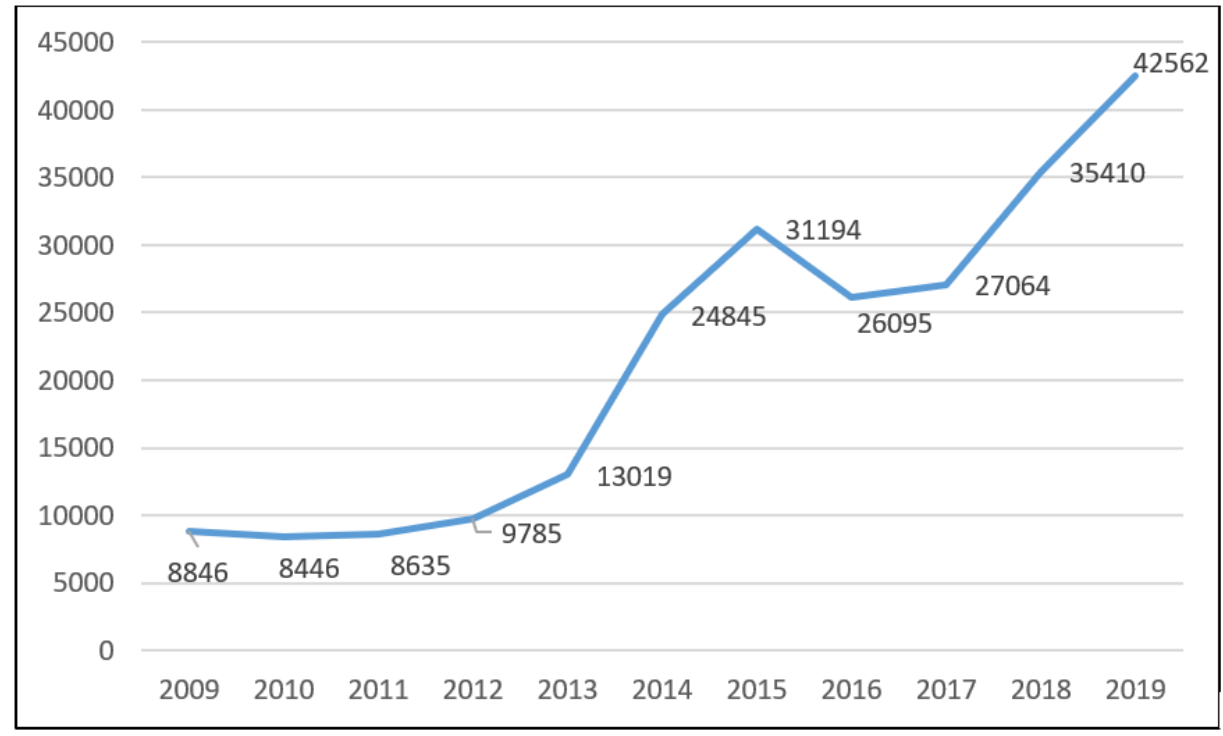

Fuente: elaboración propia a partir de LABORA (2020). Servei Valencià d'Ocupació i Formació.

“I2. Una vez los pillé por contabilidad

11. Sí ... En alguna ocasión, cuando has visitado a una empresa, pues te has encontrado, además de la factura, que está amañada, el albarán (que) sí te dice la verdad, es decir, tantos kilos a tanto el kilo, que no han llevado a la factura...

12. O porque haces una visita, tú con 7 policías nacionales, y pillas a una administrativa, que la pobre está nerviosa ... y pica donde no debiera picar" (inspectores trabajo. EG06).

Todos nuestros entrevistados y entrevistadas inmigrantes nos señalan que, al menos en sus períodos iniciales en los campos de La Ribera del Xúquer, han pasado o conocido situaciones de este tipo. Además del sueldo por debajo de convenio, la merma de días declarados y cotizados a Seguridad Social, tiene efectos negativos para la percepción del subsidio o prestación de desempleo y para la futura pensión.

"I5. Sí, cuando tú vas a cobrar el paro, después (te dicen) "es que tú apenas" y dices, pues toda la campaña de 120 días, "no, dice, si aquí apenas tienes 50, 60" dices, hostia tío, ¿Dónde está el resto de días? ... Y vas a cobrar y te quedas en la calle" (trabajador rumano, EG03).

No todos los sueldos por debajo de convenio son irregulares y deben camuflarse. Algunas empresas han utilizado la posibilidad de trabajar por debajo del convenio con el acuerdo de los representantes de los trabajadores, contemplada en la reforma laboral de 2012, para pactar acuerdos de empresa más ventajosos y perfectamente legales. 
Además de las nóminas fraudulentas, hay otra serie de irregularidades por parte de las ETT "más piratas o más sinvergüenzas" (sindicalista. El08). El o la jefe de cuadrilla obtiene su plus y, en ocasiones, su sueldo, de cobrar el transporte, de cobrar por contratar a una persona (sea mediante una cantidad fija inicial o mediante un tanto sobre los cajones recolectados), dar de alta a un trabajador inexistente o permitir trabajar a un indocumentado ${ }^{4}$.

“Ese era español el jefe de cuadrilla. Un figura... Si vienes aquí a trabajar, tú me has de dar cuatro euros todos los días. Encima, ponía a su mujer que no iba a recolectar, la ponía en la cuadrilla para que los kilos (recogidos) se repartieran en vez de catorce entre quince y ella cobraba también y no iba a trabajar. Un escándalo" (español, antiguo jornalero, sindicalista. EI08)

Según Inspección de trabajo y los sindicatos, hay muy escasas denuncias. Por cobro inferior a convenio o menos días declarados solo denuncian algunos españoles en casos graves, pero ningún inmigrante que son los más afectados por estas prácticas. Los inmigrantes sólo han denunciado por accidentes de trabajo cuando no pueden acogerse a las prestaciones.

De acuerdo con todos nuestros entrevistados, son las ETT las que concentran el mayor número de irregularidades, tanto en sueldo como en las prácticas explotadoras antes reseñadas. En expresión de uno de ellos,

“las ETT se aprovechan del estado de necesidad de los inmigrantes... hay tres ejércitos de reserva dispuestos a trabajar como sea [...] pillan al eslabón más débil de la cadena [...] que no están organizados, ni pueden [...] es muy difícil de controlar... no se puede poner puertas al campo" (español, antiguo jornalero, sindicalista. El08).

\section{ALMACENES, CALIDAD E IMPLICACIONES PARA LAS TRABAJADORAS}

En las primeras décadas del siglo XX, los almacenes eran el espacio productivo que "durante la temporada de recolección selecciona, limpia y empaqueta el fruto" (Calatayud, 1986), cuyo control estaba en manos de las compañías exportadoras de capital valenciano. Un siglo después, los almacenes de naranjas se han adaptado a las nuevas condiciones del mercado global, donde el cliente (distribuidoras y supermercados) adquiere un papel protagonista en las especificaciones del producto, los requerimientos de calidad y la fijación de los precios.

Bajo la común denominación de almacén encontramos una amplia diversidad según se trate del almacén de una empresa local, una cooperativa o de una gran distribuidora, las diferentes fórmulas de acceso al producto, sea producción propia o comprada a agricultores, su capacidad de trabajo, según instalaciones, grado de mecanización, formación del capital humano, etc. Nos referiremos a continuación a los aspectos comunes más relevantes para este artículo.

\section{El almacén como gestor de calidad}

El almacén, pese a ser formalmente el encargado del proceso de confección del producto, adquiere un papel protagonista en la materialización de los estándares de calidad, dada su posición intermedia entre los distintos agentes de la cadena (agricultores, corredores,

4 En este caso, el trabajador indocumentado ocupa el puesto de un trabajador con contrato. Su sueldo lo reparte con el jefe de cuadrilla y con el trabajador cuyos papeles utiliza. Además de indicárnoslo nuestros entrevistados, se han documentado diversos casos al producirse un accidente de trabajo. 
recolectores, clientes), una posición que asume por delegación de las grandes cadenas distribuidoras y que conlleva la asunción por su parte de riesgos y responsabilidades (como el rechazo de partidas que el cliente considere no adecuadas).

Por tanto, la gran mayoría de almacenes de cooperativas y empresas cuentan con departamentos de calidad y han contratado a personal especializado para desempeñar las tareas relacionadas con la gestión de la calidad.

De acuerdo con las y los técnicos de calidad entrevistados, los almacenes tienen al menos tres protocolos: el GlobalGAP, con el módulo GRASP de bienestar laboral, el BRC para el mercado inglés y el IFS para el mercado alemán y francés ${ }^{5}$. Además, dependiendo de clientes específicos pueden establecerse otras exigencias, tanto sobre el producto como sobre el envase y la presentación.

"Hay especificaciones de calidad de calibre, de grados brix, de índice de madurez, eso se ajusta a las normas de la Unión Europea de comercialización, creo que la 19.011, además, cada cliente tiene unas especificaciones que van más enfocadas a la calidad estética del fruto, es decir, hablamos de defectos estables y defectos inestables, los defectos estables, por ejemplo, puede ser un rameado, un defecto cosmético que no va a evolucionar, un defecto inestable, por ejemplo, pues sería un daño mecánico, que puede evolucionar a podrido... cuando manipulamos aquí en almacén le damos la capa de imazalil ¿Vale? Son ceras para evitar que cualquier daño pueda evolucionar... [...] cada cliente tiene sus envases ... hay diferentes for fates (por) su marketing, su política de mercado y demás... los protocolos ahora mismo se centran mucho en el material auxiliar de envasado, todo el tema de migración de plásticos en contacto con alimentos, entonces dificulta a veces la labor de calidad, tener tanta variedad de confecciones" (técnico calidad cooperativa, El05)

El control de calidad no se limita al almacén, sino que también se establece en campo controlando la producción. Las grandes cooperativas y empresas tienen técnicos de calidad y de campo, integrados en el mismo equipo. En muchas cooperativas es la misma persona la que realiza las funciones de técnico de calidad de almacén y de campo. Respecto a la producción, además de las exigencias del cuaderno de campo, trazabilidad y fitosanitarios, establecidas por la normativa general para todos los productores, en el caso de los agricultores tutelados se establecen exigencias mayores controladas por el técnico de calidad. Además, como hemos comentado, el jefe de cuadrilla debe "categorizar" y "valorizar" la recolección de los distintos campos para prever los tratamientos adecuados.

El punto de encuentro entre producción, recolección y confección es el muelle de descarga del almacén, punto de inicio del recorrido de confección. En primer lugar, se verifica las toneladas de fruta y el código del campo para su trazabilidad. En segundo lugar, se descarga en las lonas de selección para una primera discriminación y la línea de lavado. En tercer lugar, una vez la fruta limpia se realiza una segunda selección para detectar posibles defectos, estables e inestables. En cuarto lugar, las piezas son clasificadas por calibre y se distribuyen por las cintas correspondientes. En quinto lugar, se encaja o enmalla el producto según las especificaciones del cliente y se apilan las cajas. En sexto lugar, se prepara el pedido y se carga el camión o se mantiene en las cámaras frigoríficas hasta su envío.

Todas estas fases son controladas por los técnicos de calidad. Además, las certificaciones más importantes incluyen auditorías externas sobre la materia prima, condiciones de trabajo, higiene de las instalaciones o sobre el producto confeccionado. Las auditorías las puede realizar el propio cliente, normalmente las grandes superficies, o empresas auditoras. En los dos casos, los técnicos externos mantienen reuniones con el equipo

5 Existe una parte de almacenes de empresa que no trabajan con parámetros de calidad y se orientan a los nichos de mercancía barata, para surtir a tiendas de bajo precio, o a fábricas de zumos. 
técnico de calidad, recogen muestras del producto de distintos palets y toman fotografías.

"Hay veces que el control de calidad, vienen personas que han contratado ese producto, está repasando cajas y si no quieren no se llevan el pedido [...] Van mirando... escarbando palets. Si en ese palet le han salido muchas malas, vuelve a escarbar en otro palet. Si en el otro palet le vuelven a salir malas dice: Yo eso que habéis hecho no me lo llevo. Y hacen fotos, ¿eh?, y las envían allá (a su central). Y si allá le dicen: No, no, no. Eso no lo cargues, que no está para cargar, no hay carga. Y eso son dineros que se pierden" (española, encargada almacén cooperativa. El18)

Entre los y las técnicos de calidad, encontramos dos tipos de discursos que remiten a dos concepciones de calidad diferentes. Uno, un discurso más técnico centrado en las características del producto y los tratamientos a realizar para cumplir los requerimientos de calidad exigidos para su certificación, como ejemplifica la cita anterior. Otro, un discurso más crítico que incide en como la concepción de calidad se ha focalizado en las certificaciones y tramitación administrativa más que las propiedades intrínsecas del producto. Unos y otros discursos pueden ser utilizados por el mismo interlocutor o interlocutora.

“I1. El estándar a nivel de mercado y de superficies... se habla de calidad, pero lo menos importante es la calidad, aquí lo que estamos hablando es de certificación del producto [...] No de calidad en cuanto a parámetros organolépticos, de salud, o culinarios, o nutritivos, estamos hablando de documentación y de certificación, se construye un referencial en donde entran un montón de componentes que todos hemos asumido que forman parte del concepto amplio de calidad, pueden ser componentes ambientales, de carácter social, de carácter laboral, etcétera, y se documenta, se audita y se certifica...

12. Son estándares de diferenciación. No estándares de calidad" (entrevista técnicos calidad. EG09).

\section{La calidad y las trabajadoras de los almacenes}

La temporada de almacén tanto de cítricos como de caqui tiene una duración aproximada de seis o siete meses. La campaña suele iniciarse a finales del mes de septiembre o principios del mes de octubre y suele finalizar en los meses de marzo o abril. En algunos almacenes combinan cítricos, caqui y otras frutas con hueso, por lo que la temporada suele tener una duración superior, de ocho a diez meses prolongándose hasta mayo o junio.

En el pasado y en la actualidad, el almacén es un espacio productivo feminizado, marcado por una fuerte división sexual del trabajo. Los hombres realizan tareas que requieren mayor fuerza física (cargar y descargar camiones, apilar cajas o mover pilas de cajas) y aquellas relacionadas con la maquinaria (uso, mantenimiento). Las mujeres realizan tareas manuales que requieren destreza, rapidez y resistencia física (observación, selección y eliminación de frutos cuando es necesario, montaje de cajas, etiquetaje, encajado y envasado). A nivel organizativo, cada proceso del confeccionado está dirigido por la persona encargada de la sección que prescribe la actividad, los requerimientos de cada pedido, marca los ritmos y supervisa el trabajo de la plantilla a su cargo. La división sexual del trabajo se refleja también en los cargos intermedios, donde las mujeres suelen tener encargadas y los hombres encargados.

A diferencia del perfil de trabajador o trabajadora de recolección en el que predominan las personas inmigrantes, en el caso de los almacenes la mayoría de la plantilla es de nacionalidad española. Principalmente trabajadoras del municipio en el que está ubicado el almacén o de municipios próximos, aunque cabe destacar una paulatina incorporación 
de mujeres inmigrantes en los almacenes. Estas inmigrantes suelen ser mujeres con arraigo social y residencial en el municipio durante años y tienen una situación administrativa regularizada. En los almacenes estudiados la mayoría son mujeres de origen marroquí, del este (rumanas y búlgaras) y de forma muy minoritaria alguna latinoamericana.

"Las de almacén son casi todas del pueblo, de los pueblos de alrededor, y hay varias inmigrantes, pero que viven aquí, no son temporeras [...] Tenemos rumanas y moras. No creo que hayan más [...] Y también de Cuba varias" (española, trabajadora cooperativa. El15)

El tipo de contrato predominante en la plantilla de los almacenes es fijo discontinuo, aunque el contrato de acceso más habitual es el de trabajadora temporal. Para establecer el orden de acceso al almacén de las trabajadoras fijas discontinuas, así como para organizar los turnos de trabajo, se emplea el criterio de antigüedad reflejado en el número que posee cada trabajadora en su carné o ficha identificativa. La antigüedad se convierte en un elemento de distinción y privilegio a la hora de trabajar, ya que marca el momento de incorporación al trabajo en la temporada y la posibilidad de realizar un mayor número de horas (ordinarias y extraordinarias) ${ }^{6}$. La plantilla de trabajadoras con contrato fijo discontinuo es estable, aunque se suelen dar algunas bajas debido principalmente a oportunidades laborales en otros sectores.

Según diversas entrevistadas el número de contrataciones temporales va a la baja y aumenta el número de contrataciones eventuales vía ETT, con peores horarios y menor salario que las personas contratadas directamente por el almacén. El movimiento de las trabajadoras de ETT entre diferentes almacenes es constante durante la temporada, lo que genera escasa estabilidad laboral, un aprendizaje y adaptación constante a las tareas y organización del trabajo particulares de cada almacén y dificultad para establecer vínculos con la plantilla estable del almacén.

El salario medio de las trabajadoras contratadas por el almacén es próximo a los 1.000-1.200 euros netos mensuales por 40 horas semanales. En plena temporada y según el volumen de trabajo, se realizan horas extraordinarias entre semana y durante el fin de semana. A cada trabajadora se le añade a su nómina el computo de horas extraordinarias realizadas durante el mes, lo que puede incrementar su salario hasta 400 euros.

A diferencia de lo que ocurre en el campo, donde se constatan múltiples irregularidades, tanto los sindicalistas, las y los inspectores de trabajo, y las trabajadoras de almacén entrevistadas afirman que se cumple el Convenio Colectivo de Manipulado y Envasado de cítricos, frutas y hortalizas de almacén, en términos de salarios, horarios, etc., así como las condiciones generales de higiene, seguridad, representación de las trabajadoras y elecciones sindicales. Hay que destacar que a mediados de los años 90 se dieron luchas para dignificar la situación de las trabajadoras fijas discontinuas y que los almacenes, a diferencia del campo, es un espacio laboral con relevante presencia sindical desde hace dos décadas. Además, en los almacenes de cooperativas y de las empresas que trabajan calidad se ha generalizado el módulo GRASP, de GlobalGAP, sobre condiciones sociolaborales y exigido en la actualidad por las grandes distribuidoras y superficies.

Pese a que las condiciones laborales son percibidas como buenas en los almacenes estudiados, al preguntar sobre el impacto de los estándares de calidad sobre su trabajo las trabajadoras de almacén manifiestan incredulidad y rechazo a un supuesto aumento de calidad en sus condiciones. Cuestionan que la calidad se aplique a algo más que no sea al propio producto. Este discurso choca frontalmente con el eslogan con el que se presenta

\footnotetext{
6 En el pasado, con el primer hijo algunas trabajadoras cesaban su actividad de forma permanente, mientras otras lo hacían por uno o varios años, reincorporándose más tarde pero perdiendo la antigüedad. En la actualidad, la maternidad no constituye un punto de inflexión en el trabajo, gracias a los permisos de maternidad y a las políticas de conciliación laboral (reducción del horario de la jornada laboral, elección de turno de trabajo, etc.).
} 
la certificación GRASP en la web7 de GlobalGAP donde destaca que "las Buenas Prácticas Agrícolas no sólo se aplican a los productos, sino también a las personas".

Con el establecimiento de los estándares de calidad las trabajadoras han visto como se ha transformado su espacio de trabajo, las normas de comportamiento y el control sobre su propio cuerpo y su presentación identitaria como mujer. Las trabajadoras valoran que dichas transformaciones tienen consecuencias importantes en la organización del trabajo y en su cotidianidad. Destacan aspectos antes permitidos y ahora prohibidos como escuchar la radio, comer caramelos o chicles, tener una botella de agua o poder fumar en la puerta. Critican igualmente el uniforme obligatorio y las estrictas normas de higiene que prohíben llevar los labios pintados, pendientes... y otros aspectos de su presentación personal. Estos cambios, como señalan Moraes y Cutillas (2014) para Murcia, han tendido a disciplinar los cuerpos y resignificar el propio trabajo. Otras subrayan las restricciones de movimientos para ir al baño o a la fuente a beber o que las tareas, a diferencia del pasado, se deben realizar de pie. Este control se acentúa cuando se anuncia una auditoría, aunque tiende a ser permanente ya que algunas se realizan sin aviso previo.

"Mira, yo empecé... las mujeres mayores triando estaban sentadas. Yo me acuerdo que mi suegra estaba sentada con su capacito, con su mantita y todo. Y triaba igual que de pie [...] antes... las triadoras podían tener radio para escuchar música, eh... los anillos, pendientes no se quitaban [...] Podías ir pintada de gala, y no pasaba nada. Eh... el tiempo para ir el váter no fichabas, ... Después... todo ha cambiado con las normas europeas. Para ir a la Unión Europea tenías que cumplir X exigencias. Y ahora todas estamos de pie, música cero... chicles, caramelos, cero, estas cosas..." (polaca, trabajadora almacén. El17)

“Hace mucho tiempo que vamos de uniforme [...] No llevamos anillos, no llevamos pendientes, no puedes llevar un piercing [...] llevamos guantes, prohibido pintarte las uñas así... cantarinas, si son transparentes no se dan cuenta, pero uñas largas no quieren... dañas el producto. No es un sitio que te dejen ir maquillada" (española, trabajadora cooperativa. El15)

Por otro lado, algunas entrevistadas consideran que la incorporación de la calidad ha profesionalizado los procesos de trabajo, pero esto no conlleva una mejora de sus condiciones de trabajo e incluso añaden que comparativamente con el pasado reciente las condiciones del trabajo han empeorado, en términos de ritmo de trabajo y de clima laboral. Por un lado, la exigencia de calidad ralentiza las tareas, ya que se precisa más tiempo de observación y selección. Por otro lado, comentan la continua presión de las controladoras recordando el tipo de cliente y la exhaustividad y precisión del pedido, de forma similar a que señalan Castro et al. (2017) para la uva de mesa en Murcia. No pocas veces, sometidas a ritmos, presiones y controles constantes, se cometen errores que deben subsanarse con la saturación de trabajo consiguiente.

"La controladora de calidad, cuando vas a hacer un pedido, viene y controla si la etiqueta está bien, si la etiqueta, por casualidad, tuviera un pelillo que no sale bien, entonces si has hecho un palet, como si has hecho dos, el palet se deshace y se vuelve a hacer [...] Lo vuelvo a pasar, hay unas mujeres deshaciendo y otras mujeres poniéndolo, rápido para que cunda, o sea que siempre tienen que ir mirando que la cosa cunda... ya que te has engañado la cosa tiene que ir deprisa, muy deprisa..." (española, encargada almacén.El18)

Las irregularidades laborales, escasas, se relacionan con la falta de reconocimiento de enfermedades laborales de alta incidencia, por ejemplo, síndrome del túnel carpiano, problemas de espalda y hombros, etc., que no están recogidas, nos dicen, en el convenio. Otra queja es la utilización de las trabajadoras para la limpieza de las máquinas, tarea que precisa formación especializada o, por último, el abuso de la novena hora diaria, cobrada

7 https://www.globalgap.org/es/for-producers/globalg.a.p.-add-on/grasp/ 
a precio de hora ordinaria, que el convenio permite en momentos extraordinarios de alta producción y que, en algunos almacenes, se utiliza como prolongación sistemática y barata de la jornada laboral.

Al igual que sucedía en la recolección, las trabajadoras contratadas por ETT para los almacenes constituyen el colectivo más precarizado y presentan peores condiciones laborales. Así, el coste unitario de la hora ordinaria y extraordinaria que cobran es inferior al que cobra una trabajadora contratada por el almacén pese a realizar el mismo trabajo. Otra irregularidad que revierte también en su salario es que se les avisa para trabajar en jueves o viernes, acumulando sus horas semanales en el fin de semana para evitar pagarles horas extraordinarias pese a que trabajen el fin de semana.

“La ETT paga. Pero, igual... cobran también más bajas las horas que nosotras [...] Si por ejemplo a mí me cobran 7,5 a la hora, a ellas pagan 6. Los ETT ganan dinero de ellas [...] Las ETT para mí no debería de funcionar. Una institución de paro que busca trabajo, a ellas deberían de ir las ofertas de trabajo, no para ETT" (polaca, trabajadora almacén. El17)

Además, en algunos almacenes, a las trabajadoras de ETT se les proporciona un uniforme de papel o plástico de usar y tirar, de mala calidad, incómodo y, en algunos casos, inseguro. Se distingue así unas trabajadoras, las fijas discontinuas, de otras, las de ETT, con mayor presencia de mujeres inmigrantes, lo que es percibido por todas ellas como un elemento de segregación y una uniformización vergonzante. Una encargada se queja de ese trato.

"Suelen darles bata de papel, gorros de papel... [...] Y los guantes ya te los llevas tú [...] El babero ese de papel que las pobres parecen que vayan disfrazadas ... pobrecillas. Y la seta que se ponen en la cabeza, pobrecillas... parecen... están súper amargadas. Y te parecen que son todas iguales porque claro, las ves todas vestidas iguales. Pero... es que la cooperativa para 4 días o para 10 días... dan babero, gorro de papel" (española, encargada almacén cooperativa. El18)

\section{CONCLUSIONES}

En la actualidad, la calidad constituye un elemento central en la conformación, estratificación y funcionamiento de las cadenas globales agroalimentarias, en las que la comarca valenciana de La Ribera se encuentra plenamente inserta. Nuestros resultados muestran que las implicaciones de los estándares de calidad en estas comarcas son complejas, desiguales y no han mejorado la calidad del trabajo en general. Dichas implicaciones han contribuido a aumentar las diferencias entre los espacios sociales de trabajo que constituyen el campo y el almacén, así como a aumentar la fragmentación de los trabajadores y trabajadoras en estos espacios.

En efecto, los requerimientos de calidad en la recolección no suponen ninguna mejora en las condiciones de trabajo en el campo. Dado que la inmensa mayoría de la naranja y del caqui se recolecta a destajo, la calidad se plasma en una doble exigencia, rapidez y selección, percibiendo el mismo sueldo. Más todavía, trabajando a destajo es más probable que se comentan errores que, detectados en almacén, generan descuentos en el salario a percibir. En segundo lugar, los requerimientos de calidad, junto a otros factores, aumentan la fragmentación entre los trabajadores y trabajadoras fijos discontinuos, temporales de empresa y cooperativa, y de ETT. En tercer lugar, con la excepción de una parte de empresas y de las cooperativas, las normas de calidad son compatibles con el cúmulo de irregularidades constatado. 
El balance respecto a los almacenes es más ponderado. Por un lado, la mayor presencia sindical y la generalización del módulo GRASP de GlobalGAP, sobre condiciones sociolaborales, han constituido dos factores centrales para el cumplimiento generalizado del convenio y mejores condiciones de trabajo en comparación con las del campo. Por otro lado, las normas de calidad si bien implican una mayor profesionalización han contribuido a aumentar los requerimientos de trabajo, disciplinar el ambiente de trabajo y los cuerpos de las trabajadoras, así como resignificar su propia actividad vivida como más externa a ellas. Además, en los almacenes, como en el campo, han aumentado las diferencias entre las trabajadoras fijas discontinuas y las trabajadoras de ETT, el sector más precarizado, incluso a nivel simbólico.

Nos preguntábamos, igualmente, sobre las implicaciones de los estándares de calidad sobre la segmentación étnica del mercado de trabajo. En la recolección, los fijos discontinuos que trabajan para las empresas de calidad Premium y cooperativas suelen ser españoles, no padecen irregularidades y se cumple el convenio; las irregularidades y abusos se ceban en los trabajadores inmigrantes, en particular de ETT. En los almacenes, las trabajadoras fijas discontinuas, con antigüedad y mejores condiciones, son españolas; las trabajadoras de ETT en almacén, con peores condiciones, son inmigrantes. La distinta incidencia de la calidad en un espacio de trabajo y otro refuerza la segmentación étnica y aumenta las diferencias globales entre trabajadores de uno y otro origen.

La introducción y generalización del recurso a las ETT, en el marco establecido por las cadenas agroalimentarias, ha convertido a este agente intermediario, particularmente en el campo, en vector principal del aumento de la segmentación étnica del mercado de trabajo y de la diferenciación de condiciones según el origen, de la mayor presión sin contrapartidas sobre los trabajadores y trabajadoras en función de la calidad, y del aumento de las irregularidades laborales en el sector más indefenso, fundamentalmente inmigrantes.

El balance desde el punto de vista del sistema sexo-género en el mercado de trabajo de esta comarca es ambivalente. La división sexual del trabajo se mantiene, básicamente, en sus parámetros tradicionales, la masculinización del campo y la feminización de los almacenes. Sin embargo, si bien el trabajo en los almacenes es penoso no es menos cierto que la mayoría de las trabajadoras de almacén tienen sueldos similares a los recolectores y un indice muy inferior de irregularidades laborales.

Por último, constatamos como la calidad se conceptualiza de forma diferenciada según los distintos actores, su posición en el proceso productivo y la incidencia sobre sus condiciones de trabajo. Entre los y las técnicos de calidad, encontramos dos tipos de discursos. Uno más profesional centrado en las características del producto y los tratamientos a realizar. Otro, más crítico que destaca como la calidad se focaliza más en las certificaciones que en las propiedades intrínsecas del producto. La opinión de trabajadores y trabajadoras, sean del campo o de almacén, se pueden sintetizar afirmando que la calidad se focaliza sobre el producto, no sobre sus condiciones de trabajo.

\section{REFERENCIAS}

Barañano, M. (2005). Escalas, des/reanclajes y transnacionalismo. Complejidades de la relación global-local. En A. Ariño (comp.), Las encrucijadas de la diversidad cultural (pp. 425-451). Madrid: CIS.

Baylos, P. A. (2009). Un instrumento de regulación: empresas transnacionales y acuerdos marco globales. Cuadernos de Relaciones Laborales, 27(1), 107-146. 
Bonanno, A. y Cavalcanti, J. S. B. (2012). Globalization, Food Quality and Labor: The case of Grape Production in North-Eastern Brazil. International Journal of Sociology of Agriculture and Food, 19(1), 37-55.

Bono, E. (2010). Naranjas y desarrollo. La base exportadora de la economía del País Valenciano y el modelo de crecimiento hacia fuera. Valencia: PUV.

Caballero, P., Fernández-Zamudio, M. A., García-Martínez M. C., Carmona, B., Alcón, F. y De-Miguel, M. D. (2012). Los costes y la formación de los precios determinantes de la viabilidad de la citricultura española. Actas de Horticultura, 60, 146-151.

Calatayud Giner, S. (1986). Condiciones de trabajo en la agricultura naranjera (1914-1936). Saitabi, 36, 269-288

Carton de Grammont, H. y Lara, S. (2010). Productive Restructuring and Standardization in Mexican Horticulture: Consequences for Labour. Journal of Agrarian Change, 10 (2), 228-250.

Castro, C. de, Moraes, N. y Cutillas, I. (2017). Gobernar la producción y el trabajo por medio de estándares. El caso de la industria agroalimentaria en Murcia. Política y Sociedad, 54(1), 111-142. https://doi.org/10.5209/POSO.51494

Censo Agrario (2009). Instituto Nacional de Estadística (en línea). https://www.ine.es/CA/ Inicio.do, acceso 14 de febrero de 2020.

Cucó, J. (1982). La tierra como motivo. Jornaleros y propietarios en dos pueblos valencianos. Valencia: Institució Alfons el Magnànim.

Gallego, J. R. y Lamanthe, A. (2011). ¿Por qué las cooperativas no dominan en los sistemas hortofrutícolas tradicionales? Una comparación entre España y Francia. CIRIEC-España, Revista de Economía Pública, Social y Cooperativa, 72, 9-41.

Garrapa, A. M. (2017). The citrus fruit crisis. Value chains and just in time migrants in Rosarno (Italy) and Valencia (Spain). En A. Corrado, C. Castro y D. Perrotta (eds), Migration and Agriculture: Mobility and Change in the Mediterranean Area (pp. 111-127). London: Routledge.

Gereffi, G., Humphrey, J. y Sturgeon, T. (2005). The governance of global value chains. Review of International Political Economy, 12(1), 78-104. http://dx.doi. org/10.1080/09692290500049805

Gibbon, P., Bair, J. y Ponte, S. (2008). Governing global value chains: An introduction. Economy and Society, 37(3), 315-338. https://doi.org/10.1080/03085140802172656

Labora (2020). Servei Valencià d'Ocupació i Formació (en línea) http://www.labora.gva.es/ va/estadisticassispehtml, acceso el 5 de marzo de 2020.

Moraes, N. (2011). El impacto de los estándares de calidad y responsabilidad social en los nuevos enclaves globales agrícolas. Trabajo presentado en Jornadas Internacionales Trabajo agrícola, cadenas agroalimentarias globales y desarrollo rural, Montevideo.

Moraes, N. y Cutillas, I. (2014). Nuevos dispositivos de regulación transnacional: un análisis sobre los estándares de calidad y responsabilidad social y su impacto en los enclaves globales agrícolas. En A. Pedreño (coord.), De cadenas, migrantes y jornaleros. Los territorios rurales en las cadenas globales agroalimentarias (pp. 120-135). Madrid: Talasa Ediciones. 
Pedreño, A. (2014). Encadenados a fetiches. Del enfoque de las cadenas de mercancías a la sostenibilidad social de los enclaves de producción de la "uva global". En De cadenas, migrantes y jornaleros. Los territorios rurales en las cadenas globales agroalimentarias (pp. 13-36). Madrid: Talasa Ediciones.

Piore, M. (1983). Notas para una teoría de la estratificación del mercado de trabajo. En L. Toharia (ed.), El mercado de trabajo. Teorías y aplicaciones (pp. 193-221). Madrid: Alianza.

Ponte, S., Gibbon, P. y Vestergaard, J. (2011). Governing through Standards: An introduction. En Governing through Standards: Origins, Drivers and Limitations (pp. 1-23). Londres: Palgrave MacMillan.

Pries, L. (2008). La emergencia de un tejido de regulación laboral transnacional. Sociología del Trabajo, 63, 27-48.

Quaranta, G. (2009). Organización y mercados de trabajo en la producción de uva para el consumo en fresco en la provincia de San Juan. Trabajo presentado en Seminario taller Migraciones y calidad del empleo agrícola, Neuquén, Argentina.

Robertson, R. (1995). Glocalization: Time-Space and Homogeneity-Heterogeneity. En M. Featherstone, S. Lash y R. Robertson (eds.), Global Modernities (pp. 25-44). London: Sage Publications.

Selwyn, B. (2013). Social Upgrading and Labour in Global Production Networks: A critique and an alternative conception. Competition and Change, 17(1), 75-90. https://doi.org/10. $1179 / 1024529412 Z .00000000026$

Striffer, S. (2008). El fruto del neoliberalismo. Organización laboral transnacional en el contexto de la industria bananera global y el caso ecuatoriano. Clio América, 2(4), 179194.

Vendrell, M. (2017). El milagro del caqui en Valencia. Un caso de innovación abierta y colaborativa de liderazgo cooperativo (Trabajo fin de Máster). Universidad Politécnica de Valencia, Departamento de Economía y Ciencias Sociales, Máster Universitario en Economía Agroalimentaria y del Medio Ambiente, Valencia.

\section{NOTA BIOGRÁFICA}

Francisco Torres Pérez es Profesor Titular de Sociología en la Universidad de Valencia. Sus campos de investigación son la sociología de las migraciones y la sociología urbana con diversas líneas como la inserción laboral y vecinal, en ámbitos rurales y urbanos, sociabilidad y relaciones inter-étnicas, políticas públicas de inmigración y urbanismo neoliberal y desigualdad urbana.

Yaiza Pérez Alonso, licenciada en Sociología y posgraduada en el Máster Internacional de Migraciones por la Universitat de València y en el Posgrado de especialistas en Investigación Social Aplicada y análisis de datos del CIS. Profesora asociada al Departamento de Sociología y Antropología Social de la Universitat de València e investigadora en Eixam Estudis Sociològics SLU. Sus líneas de investigación están relacionadas con las migraciones, las condiciones laborales de los y las inmigrantes y la diversidad etnocultural. 